\title{
Epigallocatechin gallate attenuates overload-induced cardiac ECM remodeling via restoring $T$ cell homeostasis
}

\author{
YONGSHENG HAN $^{1}$, QINGTONG WANG ${ }^{2}$, XIZHEN FAN $^{1}$, JUN CHU ${ }^{3}$, JUNFU PENG $^{1}$, \\ YOUSHENG ZHU ${ }^{1}$, YAN LI ${ }^{1}$, XIAOJING LI ${ }^{1}$, LEI SHEN ${ }^{1}$, JAMES ASENSO $^{2}$ and SHANFENG LI ${ }^{1}$ \\ ${ }^{1}$ Emergency Center, Affiliated Anhui Provincial Hospital, Anhui Medical University, Hefei, Anhui 230001; \\ ${ }^{2}$ Institute of Clinical Pharmacology, Anhui Medical University, Key Laboratory of Anti-Inflammatory and \\ Immune Medicine, Ministry of Education, Hefei, Anhui 230032; ${ }^{3}$ Department of Cardiology, Affiliated \\ Anhui Provincial Hospital, Anhui Medical University, Hefei, Anhui 230001, P.R. China
}

Received February 3, 2017; Accepted July 11, 2017

DOI: $10.3892 / \mathrm{mmr} .2017 .7018$

\begin{abstract}
It has previously been demonstrated that Epigallocatechin gallate (EGCG) has regulatory effects on cellular immunity. The present study explored whether EGCG inhibits the overload-induced cardiac extracellular matrix (ECM) remodeling through targeting the balance of $\mathrm{T}$ cell subpopulations. Sprague-Dawley rats were subjected to either transverse aortic constriction (TAC) or sham operation. TAC rats were treated with EGCG or valsartan (Val) for 6 weeks. The administration of EGCG or Val ameliorated the overproduction of cardiac collagen, inhibited matrix metalloproteinase (MMP) activity, decreased the expression of tissue inhibitor of MMP-2, atrial natriuretic peptide and brain natriuretic peptide. EGCG regulated the population of effector $\mathrm{T}$ cells and naïve $\mathrm{T}$ cells, restored the balance of $\mathrm{T}$ helper (Th) cell 17/regulatory $\mathrm{T}$ cells, via modulating the downstream regulator signal transducer and activator of transcription (STAT3) and STAT5. Furthermore, the ratio of interferon- $\gamma /$ interleukin (IL)-10 which indicates the balance of Th1/Th2, was restored by the treatments at varying degrees. EGCG and Val administration rescued IL-7 production, and decreased the level of IL-15 in TAC rats. EGCG has positive therapeutic potential in inhibiting cardiac ECM remodeling. Regulation of the balance of $\mathrm{T}$ lymphocyte subsets may be one of the underlying mechanisms responsible for this effect.
\end{abstract}

\section{Introduction}

Cardiac extracellular matrix (ECM) remodeling is the most severe clinical manifestations during the process of heart

Correspondence to: Dr Qingtong Wang, Institute of Clinical Pharmacology, Anhui Medical University, Key Laboratory of Anti-Inflammatory and Immune Medicine, Ministry of Education, 81 Meishan Road, Hefei, Anhui 230032, P.R. China

E-mail: hfwqt727@163.com

Key words: epigallocatechin gallate, cardiac remodeling, $\mathrm{T}$ helper cell 17 , regulatory $\mathrm{T}$ cells, immunological regulation failure (HF), which is characterized by myocytes hypertrophy, excessive expansion and accumulation of ECM, less compliance, and matrix metalloproteinases (MMPs) activation (1). Inflammation is evidently involved in adverse cardiac remodeling and HF. Accumulating evidences have shown that the elevated levels of pro-inflammatory cytokines including interleukin-6 (IL-6) and IL-17, are significantly correlated with the development of HF, especially with the ECM deposit (2). Chronic IL-6 stimulation results in hypertrophy and dysfunction of left ventricular (LV), accordingly, depletion of IL-6 could effectively prevent LV hypertrophy induced by the infusion of angiotensin II (AngII) (3). Besides the production of cytokines, the imbalance of the $\mathrm{CD}^{+} \mathrm{T}$-helper (Th) lymphocytes subtypes Th1/Th2, later the imbalance of Th17/regulatory $\mathrm{T}$ (Treg) has been reported in the inflammatory microenvironment. The ratios of T-bet/GATA- 3 and IFN- $\gamma / \mathrm{IL}-4$ were markedly up-regulated in C57BL/6 mice treated with alcohol than that of the control group, suggesting that activating a Th2-type immune response is one of the underlying mechanisms of alcohol-induced cardiac remodeling (4). Treatments that raise the expression of IL-10, a Th2 cytokine, have been reported to be a promising therapeutic strategy to prevent the progress of pressure overload-induced adverse cardiovascular remodeling (5). Specifically, T lymphocytes were accepted to play a critical role both in the noncardiac tissues remodeling processes, and cardiac ECM remodeling and HF. Therefore, medications effectively control inflammation and $\mathrm{T}$ cell balance in the heart may provide therapeutic benefits (6).

Green tea was used to treat various diseases in the traditional Chinese medicine. Later people realized that Epigallocatechin gallate (EGCG) is the major catechin in green tea and is regarded to have strong antioxidant activity, which may prevent cell damage, exert numerous protective effects on cancer growth, hyperlipidemia, diabetes, stroke, particularly on the cardiovascular system (7-9). Green tea consumers have lower death risk from cardiovascular diseases. A recent meta-analysis of 18 published studies revealed that the mortality of cardiovascular diseases decreased by $5 \%$ if the patients' green tea consumption increased one cup per day (10). Long-term consumption of green tea may reduce the risk of attack and slightly drop the systolic and diastolic blood 
pressures $(11,12)$. Consequently, EGCG has been accepted as a potential novel approach for treating various cardiovascular diseases (13). We reported recently that EGCG markedly inhibited the viability, proliferation and collagen production of myocytes induced by AngII (14). EGCG may regulate cellular immunity as reported that EGCG could target Th cells and natural killer cell (15). Thus, we hypothesized that EGCG inhibits the overload-induced ECM remodeling partially through regulating $\mathrm{T}$ cell subtypes and corresponding pro-inflammatory cytokines.

\section{Materials and methods}

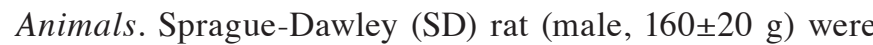
provided by the Experimental Animal Department of Anhui Medical University, China (certificate no. SCXK [WAN] 2011-002). All rats were fed in an SPF environment. All experiments were approved by the Ethics Review Committee for Animal Experimentation of Anhui Medical University and were conducted according to the Declaration of Helsinki.

Materials and drugs. EGCG and AngII receptor inverse agonist valsartan (Val) were both purchased from Sigma Chemical Co. (St. Louis, MO, USA). Anti-rat CD4- FITC, CD3- PE, IL-17-PE, CD44-PE, CD62L-PE, PE isotype, CD25-PE antibodies, anti-rat/mouse Foxp3-PECy5 antibody and PECy5 isotype were the products of eBioscience, Inc. (San Diego, CA, USA) or BD Biosciences. Phorbol myristate acetate (PMA) and ionomycin were purchased from Sigma-Aldrich (St. Louis, MO, USA). ELISA kits, anti-rat signal transducer and activator of transcription 3 (STAT3) and anti-rat STAT5 were obtained from R\&D Systems, Inc., Minneapolis, MN, USA. MMPs activity assay kit (Fluorometric-Red; Abcam, Hong Kong SAR, China).

Establishment of pressure overload-induced cardiac remodeling model and treatment. SD rats were randomly subjected to either transverse aortic constriction (TAC, to induce chronic pressure overload) or sham operation following the protocol described previously (16). Briefly, the rats were anesthetized and the chest was opened, the intercostal muscles were then blunt dissected and followed by the identification of thoracic aorta, a 6.0 silk suture was placed around the transverse aorta, and a 26-gauge blunt needle was put parallel to the transverse aorta. To make a constriction, the needle was gently removed after the suture was tied. Sham-operated rats $(\mathrm{n}=10)$ underwent a similar surgical procedure without the ligation. TAC rats were randomly divided into a model group which was treated with vehicle; three EGCG-treatment groups (orally fed EGCG 25, 50 or $100 \mathrm{mg} / \mathrm{kg} \mathrm{B.W}$. day for 6 weeks) and Val-treatment group (orally fed Val $30 \mathrm{mg} / \mathrm{kg} \mathrm{B.W}$. day for 6 weeks) one week after the surgery.

Masson's Trichrome staining of heart tissue. The hearts of rats were perfused with PBS and fixed in formalin and then were embedded in paraffin and sectioned into five-micron slices using Thermo HM355 Microtomes. The Masson's Trichrome staining was performed according to the instruction. The blue-stained areas in the sections were measured with Image J software (NIH, Bethesda, MD, USA) to evaluate the collagen deposition semi-quantitatively. The data from six regions of each heart was analyzed.

Determination of cardiac ECM. The micrograms of collagen per milligram of the dry heart were used to reflect the extent of cardiac ECM deposition. Since collagen contains about $13.5 \%$ hydroxyproline (17), the level of total myocardial hydroxyproline was determined with the standard trans-hydroxyproline colorimetric curve (Sigma-Aldrich).

Analysis of $C D 4^{+} C D 44^{+}$and $C D 4^{+} C D 62 L^{+} T$ cells. Splenic lymphocytes were isolated by density gradient centrifugation from splenic cells and then were stained with CD4-FITC and CD44-PE, or CD4-FITC and CD62L-PE respectively. The percentage of $\mathrm{CD} 4{ }^{+} \mathrm{CD} 44^{+}$and $\mathrm{CD} 4{ }^{+} \mathrm{CD} 62 \mathrm{~L}^{+} \mathrm{T}$ cells were analyzed by flow cytometry on an FC500 flow cytometer (Beckman Coulter, Fullerton, CA, USA). Data analysis was performed using Cell Quest ${ }^{\mathrm{TM}}$ analysis software.

Detection of Th17 cells. Splenic lyomphocytes were suspended in DMEM medium at a concentration of $1 \times 10^{7}$ cells $/ \mathrm{ml}$ and were stimulated with PMA (final concentration is $50 \mathrm{ng} / \mathrm{ml}$ ) and ionomycin (final concentration is $1 \mu \mathrm{M}$ ) for $5 \mathrm{~h}$ in the incubator. Golgistop (final concentration is $0.7 \mu \mathrm{l} / \mathrm{ml}$ ) was added $1 \mathrm{~h}$ after culture. The subpopulation of Th17 (CD4 $\left.{ }^{+} \mathrm{IL}-17^{+}\right)$ cells was counted on an FC500 flow cytometer (Beckman Coulter) (18).

Determination of regulatory $T$ cells (Tregs). Isolated splenic lymphocytes $(100 \mu \mathrm{l})\left(1 \times 10^{7}\right.$ cells $\left./ \mathrm{ml}\right)$ were stained with $0.125 \mu \mathrm{g}$ anti-rat CD4-FITC and $0.06 \mu \mathrm{g}$ anti-rat CD25-PE antibodies in the dark at $4^{\circ} \mathrm{C}$ for $30 \mathrm{~min}$. The cells were then fixed and permeabilized, blocked with $2 \%$ normal rat serum. $0.5 \mu \mathrm{g}$ anti-mouse/rat Foxp3-PeCy5 antibody or rat $\operatorname{IgG} 2 \mathrm{a}$ $\mathrm{K}$ isotype control PeCy5 was added to each sample without washing after the blocking step and incubated at $4{ }^{\circ} \mathrm{C}$ for $30 \mathrm{~min}$ in the dark. 100,000 cells were counted on FC500 flow cytometer (Beckman Coulter) (19).

Determination of MMP activity. The activity of MMP was evaluated with an MMP activity assay kit. Briefly, $10 \mathrm{mg}$ heart tissue was homogenized in $1 \mathrm{ml}$ extraction buffer. Samples were spined and the supernatants were then incubated with equal volume of $2 \mathrm{mM}$ p-Aminophenylmercuric acetate working (2x) solution for $15 \mathrm{~min}$, then incubated with $50 \mu \mathrm{l}$ MMP red substrate solution for $0 \mathrm{~min}$ (for kinetic reading) or $1 \mathrm{~h}$ (for end point reading), the fluorescence intensity was monitored with absorbance reader (ELx808, BioTek, US) and the MMP activity was analyzed as Ex/Em=540/590 nm.

The expression of TIMP-2, ANP, BNP, T-bet, GATA-3, RORC and FoxP3 mRNA in heart tissue. The total RNA of splenic lymphocytes was extracted using the traditional TRIzol method. The primers used for qPCR are listed in Table I (20).

Determination of serum $I L-6, I L-7, I L-15, I L-17, I F N-\gamma$, and $I L-10$. Blood was centrifuged at $3000 \times \mathrm{g}$ for $30 \mathrm{~min}$ at $4^{\circ} \mathrm{C}$, and the serum was collected. IL-6, IL-7, IL-15, IL-17, IFN- $\gamma$ and IL-10 levels were measured using ELISA kits. Absorbance 
Table I. Primer sequences for qPCR.

\begin{tabular}{lcc}
\hline Gene & Primer & Sequences \\
\hline FoxP3 & F & 5'-TGAGCTGGCTGCAATTCTGG-3' \\
& R & 5'-ATCTAGCTGCTCTGCATGAGGTGA-3' \\
RORC & F & 5'-GGATGAGATTGCC CTCTACAC-3' \\
& R & 5'-GGAGGCCTTGTCGATGAGTC-3' \\
T-bet & F & 5'-AACCAGTATCCTG TTCCCAGC-3' \\
& R & 5'-TGTCGCCACTGGAAGGATA G-3' \\
GATA-3 & F & 5'-CTCTCCTTTGCTCACCTTTTC-3' \\
& R & 5'-AAGA GATGCGGACTGGAGTG-3' \\
TIMP-2 & F & 5'-GGATTCCGGGAATGACATCTAT-3' \\
& R & 5'-CGCCTTCCCTGCAATTAGATA-3' \\
ANP & F & 5'-GAGGAGAAGATGCCGGTAG-3' \\
& R & 5'-CTAGAGAGGGAGCTAAGTG-3' \\
BNP & F & 5'- TGATTCTGCTCCTGCTTTTC-3' \\
& R & 5'-GTGGATTGTTCTGGAGACTG-3' \\
GAPDH & F & 5'-TCAAGAAGGTGGTGAAGCAG-3' \\
& R & 5'-AGGTGGAAGAATGGGAGTTG-3'
\end{tabular}

values were read at $450 \mathrm{~nm}$ with an ELISA plate reader (ELx808; BioTek, Winooski, VT, USA) (21).

Western blot analysis. Purified splenic lymphocytes were applied for western blot assay to detect the expression level of STAT3 and STAT5 following the conventional method. Briefly, the cells were lysed with RIPA buffer and the proteins were separated in a running gel. After the proteins had been transferred onto a PVDF membrane, the membrane was blocked in $5 \%$ fat-free milk and stained with anti-STAT3, anti-STAT5 or anti- $\beta$-actin primary antibody in $4^{\circ} \mathrm{C}$ overnight, followed by the staining of secondary antibody. The membranes were then scanned using an imaging densitometer (GS-700; Bio-Rad, Berkeley, CA, USA). The images were quantified with ImageJ software (NIH).

Statistical analysis. At least triplicate determinations were made for each experiment. The representative results were shown in indicated cases. All data are expressed as the mean and standard deviation (SD). For multiple group comparisons, we used ANOVA and Tukey's post test. $\mathrm{P}<0.05$ was considered to indicate a statistically significant difference.

\section{Results}

The effect of EGCG on cardiac fibrosis. Masson's Trichrome staining was applied to demonstrate the cardiac fibrosis and ECM deposition. As expected, EGCG $100 \mathrm{mg} / \mathrm{kg}$ or Val $30 \mathrm{mg} / \mathrm{kg}$ treatment effectively ameliorated the development of ventricular fibrosis (Fig. 1A). The data was semi-quantified in Fig. 1B.

Collagen is a major component of the cardiac ECM, the amount of cardiac fiber detected by the determination of the total amount of hydroxyproline represents the expression level of collagen. As shown in Fig. 1C, the collagen contents in heart tissues from TAC rats were increased significantly compared with that of the sham-operated rats, the administration of EGCG $(100 \mathrm{mg} / \mathrm{kg})$ or Val $(30 \mathrm{mg} / \mathrm{kg})$ decreased total collagen content in overload-induced cardiac remodeling rats with various degrees. This result confirmed that EGCG is an effective preventer to against cardiac ECM accumulation. The mechanism is not clearly understood.

As shown in Fig. 1D, the MMPs activity in the cardiac tissue of overload-induced cardiac remodeling rats was evoked remarkably. The administration of EGCG to rats with TAC dose-dependently blocked the activity of MMPs. Val treatment slightly attenuated the increment of MMPs activity $(\mathrm{P}=0.038)$. The tissue inhibitor of metalloproteinases (TIMPs) specifically inhibit MMPs, we detected the mRNA level of TIMP-2 in heart tissue by qRT-PCR. TIMP-2 mRNA expression increased by 2.74 fold in TAC rats than that of normal rats. EGCG 50 and $100 \mathrm{mg} / \mathrm{kg}$ treatment, as well as Val administration significantly reduced the expression of TIMP-2 (Fig. 1E).

Besides excessive expansion and accumulation of ECM, MMPs activation, ECM remodeling is also characterized by myocytes hypertrophy. ANP and BNP are common hypertrophic markers. As shown in Fig. 1F and G, ANP (2.503 \pm 0.456$)$ and BNP $(1.765 \pm 0.216)$ gene expression of the TAC rats was significantly upregulated, EGCG 50 and $100 \mathrm{mg} / \mathrm{kg}$ treatment and Val $30 \mathrm{mg} / \mathrm{kg}$ treatment markedly reduced both ANP and BNP expression.

The effect of EGCG on T cell activation. We found that T cells were activated and differentiated into $\mathrm{CD} 4{ }^{+} \mathrm{CD} 44^{+}$effector $\mathrm{T}$ cells (Teff) in splenic lymphocytes from TAC rats, meanwhile, the population of $\mathrm{CD}^{+}{ }^{+} \mathrm{CD} 62 \mathrm{~L}^{+}$naïve $\mathrm{T}$ cells (Tnaive) was diminished. EGCG $(50,100 \mathrm{mg} / \mathrm{kg})$ treatment decreased the percentage of Teff subset and EGCG $(100 \mathrm{mg} / \mathrm{kg})$ rescued the subpopulation of Tnaive (Fig. 2A and B). As a result, EGCG restored the balance of Teff/Tnaive (Fig. 2C), suggesting that it effectively inhibited $\mathrm{T}$ cells activation.

The effect of EGCG on the percentage of Th17 and Treg cells. Th17 cells were identified by expression of intracellular IL-17 and surface marker CD4 from rat spleens by flow cytometry (22). It turned out that the percentages of Th17 cells represented a much higher value in overload-induced cardiac remodeling rats $(13.4 \%)$ compared with the sham-operated rats $(0.98 \%)$. As expected, Th17 subpopulation was substantially reduced in EGCG (50 mg/kg) (7.77\%), EGCG $(100 \mathrm{mg} / \mathrm{kg})(3.32 \%)$ or Val $(30 \mathrm{mg} / \mathrm{kg})(5.63 \%)$ treatment groups (Fig. 3A).

We next counted the subpopulation of Treg subsets by identifying the expression of nucleic transcriptional factor Foxp3 in $\mathrm{CD}^{+} \mathrm{CD} 25^{+}$rat splenic lymphocytes. Unlike the changes of Th17, the percentage of Treg cells was decreased obviously in rats with overload-induced cardiac remodeling $(0.59 \pm 0.15 \%)$ compared with control rats $(2.15 \pm 0.39 \%)$. The administration of EGCG $(50 \mathrm{mg} / \mathrm{kg})$ restored the content of Treg to $1.22 \pm 0.36 \%$. In particular, the administration of EGCG $(100 \mathrm{mg} / \mathrm{kg})$ significantly increased the percentage of Treg cells $(2.35 \pm 0.48 \%)$. Val exerted a similar effect as EGCG (1.29 $\pm 0.28 \%, P=0.021)$ (Fig. 3B). Comparingwith the sham rats, TAC group exhibited an increase in the ratio of Th17/Treg. EGCG (50, $100 \mathrm{mg} / \mathrm{kg})$ and Val $(30 \mathrm{mg} / \mathrm{kg})$ 
A

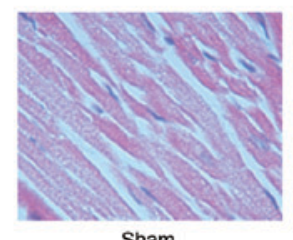

Sham

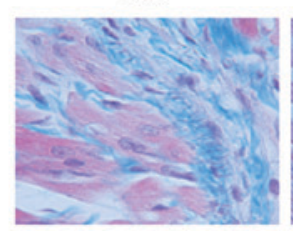

$\mathrm{TAC}+\mathrm{EGCG} 50 \mathrm{mg} / \mathrm{kg}$

C

$$
\begin{aligned}
& \square \text { Sham } \\
& \square \text { TAC } \\
& \square \text { TAC+EGCG } 25 \mathrm{mg} / \mathrm{kg} \\
& \square T A C+E G C G 50 \mathrm{mg} / \mathrm{kg} \\
& \square T A C+E G C G 100 \mathrm{mg} / \mathrm{kg} \\
& \square \text { TAC+Val } 30 \mathrm{mg} / \mathrm{kg}
\end{aligned}
$$

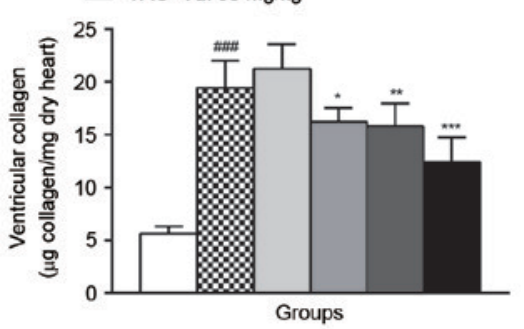

$\mathrm{F}$

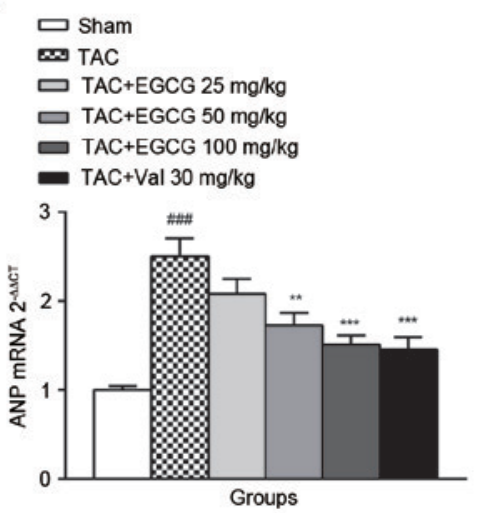

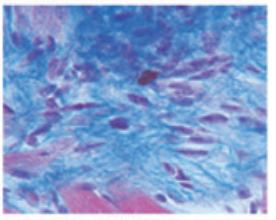

TAC

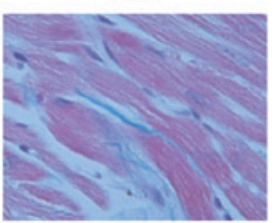

TAC+EGCG $100 \mathrm{mg} / \mathrm{kg}$

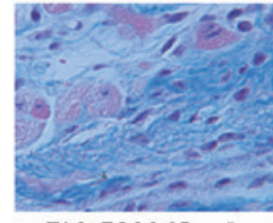

TAC+EGCG $25 \mathrm{mg} / \mathrm{kg}$

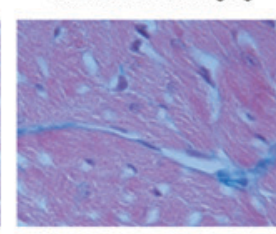

TAC+Val $30 \mathrm{mg} / \mathrm{kg}$

D

\section{$\square$ Sham}

$\infty \begin{array}{cc}\text { TAC } \\ \text { TAC }\end{array}$

$\square$ TAC+EGCG $25 \mathrm{mg} / \mathrm{kg}$

च TAC+EGCG $50 \mathrm{mg} / \mathrm{kg}$

- TAC+EGCG $100 \mathrm{mg} / \mathrm{kg}$

- TAC+Val $30 \mathrm{mg} / \mathrm{kg}$

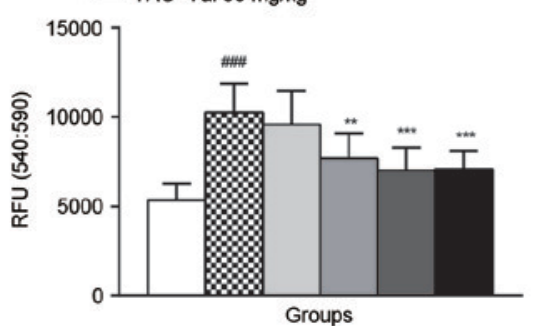

G

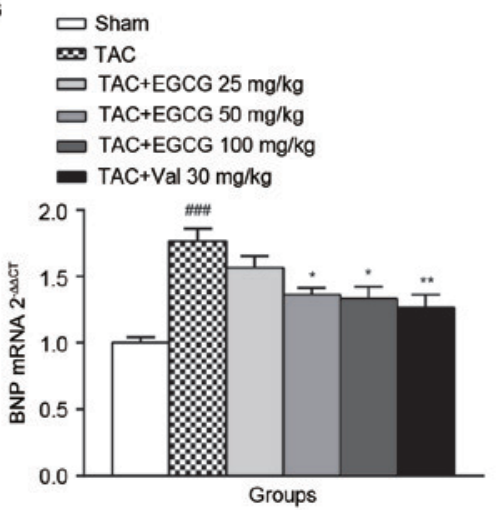

$$
\begin{aligned}
& \square \text { Sham } \\
& \varpi \text { TAC } \\
& \square \text { TAC+EGCG } 25 \mathrm{mg} / \mathrm{kg} \\
& \square \text { TAC+EGCG } 50 \mathrm{mg} / \mathrm{kg} \\
& \square \text { TAC+EGCG } 100 \mathrm{mg} / \mathrm{kg} \\
& \text { TAC+Val } 30 \mathrm{mg} / \mathrm{kg}
\end{aligned}
$$

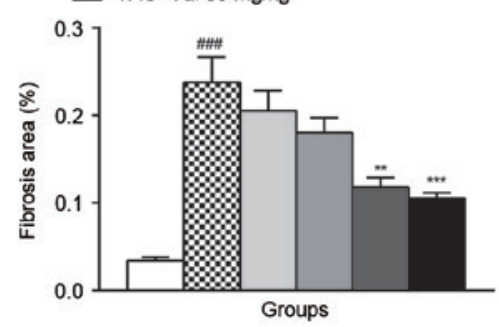

E

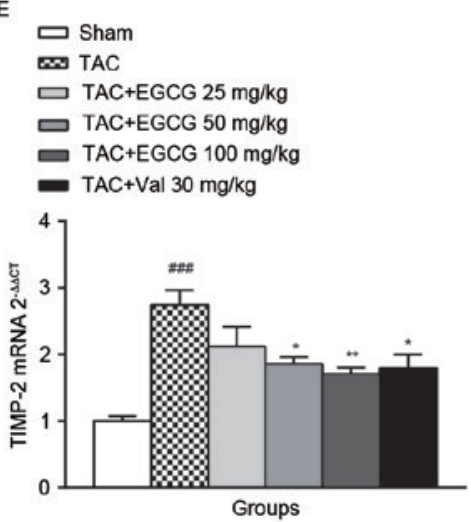

Figure 1. The effect of EGCG on cardiac fibrosis. (A) representative Masson's trichrome staining sections for all experimental groups respectively, the regions stained blue indicate collagen deposition $(\mathrm{n}=8)$. (B) Quantification of the relative area of interstitial collagen by Image $\mathrm{J}$ program. (C) Total cardiac fibrillar collagen was assayed by determination of the hydroxyproline concentrations, assuming that collagen contains an average of $13.5 \%$ hydroxyproline ( $\mathrm{n}=8-10$ ). (D) The fluorescence signal of the activity of MMPs was monitored one hour after the start of the reaction by using a microplate reader with a filter set of Ex/Em=540/590 nm (n=8). The mRNA expression of cardiac TIMP-2 (E), ANP (F), and BNP (G) was analyzed by qRT-PCR, and the gene levels were analyzed by $2^{-\triangle \Delta C q}$ and normalized to GAPDH. ${ }^{\# \#} \mathrm{P}<0.001$ vs. sham-operated group; ${ }^{*} \mathrm{P}<0.05,{ }^{* *} \mathrm{P}<0.01,{ }^{* * *} \mathrm{P}<0.001$ vs. TAC group. EGCG, Epigallocatechin gallate; MMP, matrix metalloproteinase; TIMP, tissue inhibitor of metalloproteinase.

restored the imbalance of Th17/Treg ratio significantly (Fig. 3C).

The effect of EGCG on the expressions of regulators for Th17 and Treg cells differentiation. Besides the percentage determination, we further analyzed the expressions of transcription factor RORC for Th17 and FoxP3 for Treg cells, respectively. RORC encoding retinoid-related orphan receptor $\gamma \mathrm{t}(\mathrm{ROR} \gamma \mathrm{t})$ which is identified as the master transcription factor designating Th17 cell subpopulation. In accordance with the results of flow cytometry, we found RORC was upregulated in splenic lymphocytes from TAC rats, EGCG treatment restored its expression in a dose-dependent manner (Fig. 4A). The polarization and maturation of Treg are tightly controlled by FoxP3, which expression was shown to be inhibited in the TAC group, EGCG and Val significantly rescued FoxP3 expression (Fig. 4B). The differentiation of Th17 and Treg cells is mediated by downstream regulator STAT3 and STAT5 respectively (23). As expected, the expression of STAT3 was substantially increased and STAT5 was obviously decreased in splenic lymphocytes of TAC mice. The administration of EGCG and Val was found to have distinct restorative effects on the expression of both regulators (Fig. 4C and D). 
A

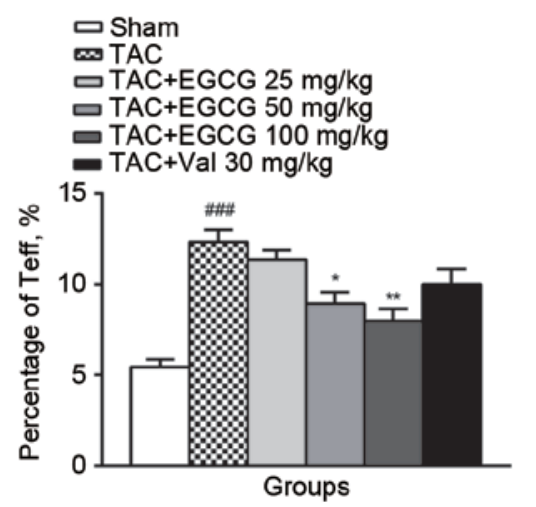

B

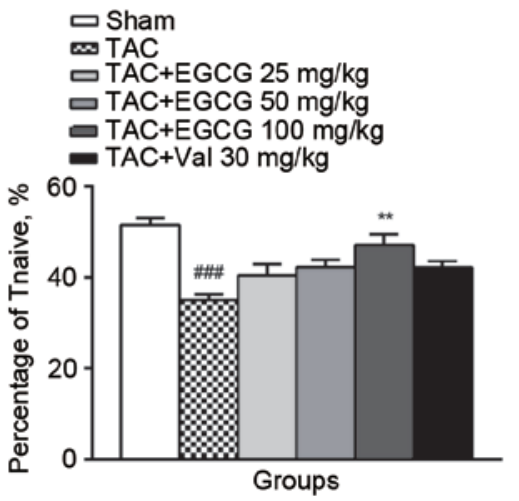

C

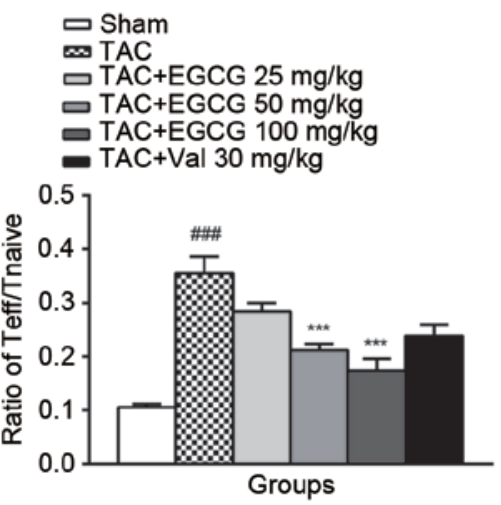

Figure 2. The effect of EGCG on the population of effector and naive T cells. (A) Histogram of the percentage of CD4 ${ }^{+} \mathrm{CD} 44^{+}$Teff ( $\mathrm{n}=8$ ). (B) Histogram of the percentage of $\mathrm{CD}^{+}{ }^{+} \mathrm{CD} 62 \mathrm{~L}^{+}$Tnaive $(\mathrm{n}=8)$. (C) The ratio of Teff/Tnaive in all groups. ${ }^{\# \# *} \mathrm{P}<0.001$ vs. sham-operated group; ${ }^{*} \mathrm{P}<0.05,{ }^{* * *} \mathrm{P}<0.01,{ }^{* * * *} \mathrm{P}<0.001$ vs. TAC group. EGCG, Epigallocatechin gallate; TAC, transverse aortic constriction.

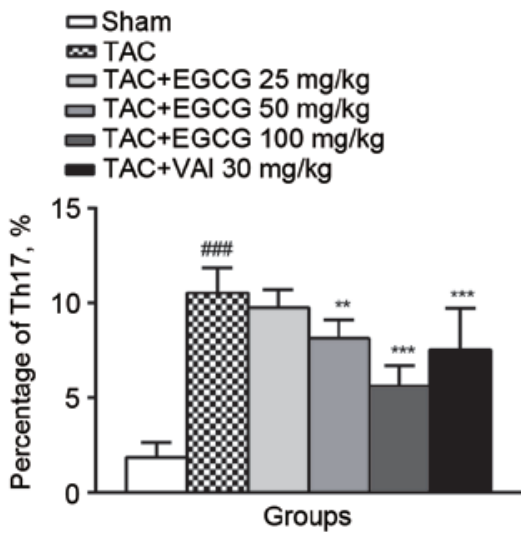

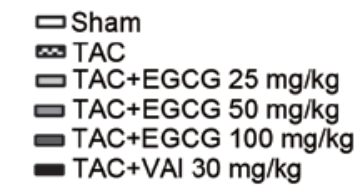

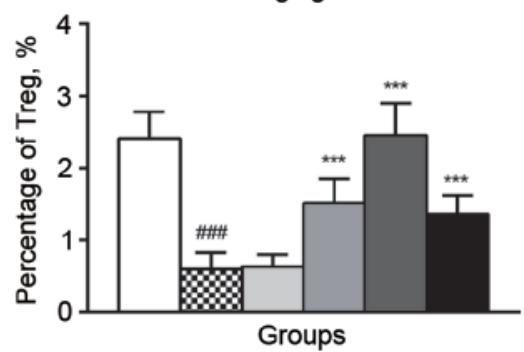

C
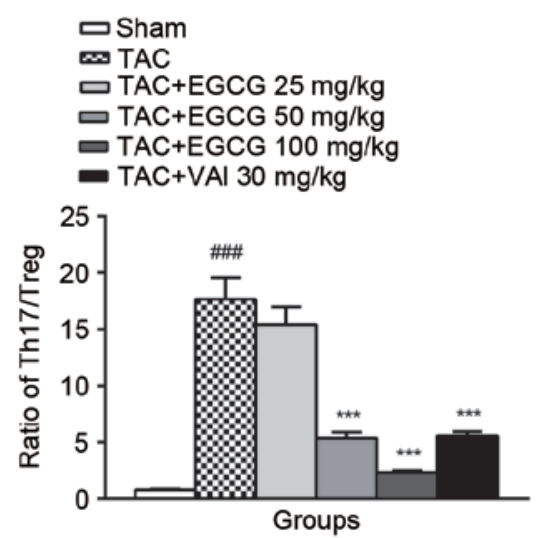

Figure 3. The effect of EGCG on the percentage of Th17 and Treg cells. (A) Quantification of the relative percentage of Th17 cells ( $\mathrm{n}=8$ ). (B) Quantification of the relative percentage of Treg cells $(n=8)$. (C) The ratio of Th17/Treg in all groups. ${ }^{\# \# \#} \mathrm{P}<0.001$ vs. sham-operated group; ${ }^{* *} \mathrm{P}<0.01,{ }^{* * *} \mathrm{P}<0.001$ vs. TAC group. EGCG, Epigallocatechin gallate; TAC, transverse aortic constriction.

The effect of EGCG on the levels of serum Th1/Th2 cytokines. As shown in Fig. 5A, IL-7 expression was obviously reduced, while IL-15 level was increased in TAC rats comparing to that of normal rats. EGCG and Val administration restored IL-7 production, and decreased the level of IL-15 in TAC rats at different degrees. The concentrations of serum IL-6, IL-17, and IFN- $\gamma$ were significantly elevated (Fig. 5A and B), while serum IL-10 was decreased in TAC rats (Fig. 5C). EGCG $(50 \mathrm{mg} / \mathrm{kg})$ inhibited the upregulation of IL-6 and IFN- $\gamma$, EGCG $(100 \mathrm{mg} / \mathrm{kg})$ reduced the production of IL-6, IL-17 and IFN- $\gamma$, and the IL-10 synthesis was restored to almost the normal level. Similarly, Val treatment modulated the levels of serum IL-6, IL-17, IFN- $\gamma$ and IL-10 with varying degrees. These data indicated that rats with overload-induced cardiac remodeling have more Th1 cytokine IFN- $\gamma$ and less Th2 cytokine IL-10. Administration of EGCG or Val ameliorated the disequilibrium of IFN- $\gamma /$ IL-10 ratio (Fig. 5D), which suggests an imbalanced ratio of Th1/Th2 subsets, consequently improved disease symptoms.

Both Th1 and Th2 populations could be differentiated from naive Th cells, each of them has distinct functions and cytokine profiles (24). Comparing with sham-operated control, the mRNA levels of T-bet, a T-box transcription factor required for Th1 cell development, in splenic lymphocytes from TAC rats was significantly increased $\left(2^{-\Delta \Delta C q}=18.34\right)$. The treatment of EGCG $(100 \mathrm{mg} / \mathrm{kg})$ or Val $(30 \mathrm{mg} / \mathrm{kg})$ mildly decreased T-bet gene expressions (Fig. 5E). However, the level of GATA-3 mRNA, a critical regulator of Th2 polarization, was decreased in model rats. Oral administered EGCG (50, $100 \mathrm{mg} / \mathrm{kg}$ ) or Val to TAC rats positively changed the GATA-3 mRNA expression (Fig. 5F). In TAC model rats, the ratio of T-bet/GATA-3 was found to be elevated, which could be balanced by EGCG treatment (Fig. 5G).

\section{Discussion}

In accordance with published articles, we observed that the collagen was accumulated in the heart of TAC rats, the MMPs were also found to be activated significantly, MMPs inhibitor TIMP-2 was induced simultaneously. The ECM may expand under microenvironmental stimuli including inflammatory cytokines (25). The increased cardiac collagen expression paralleled to the ventricular stiffness was described by Badenhorst et al (26). As expected, heart failure 
A

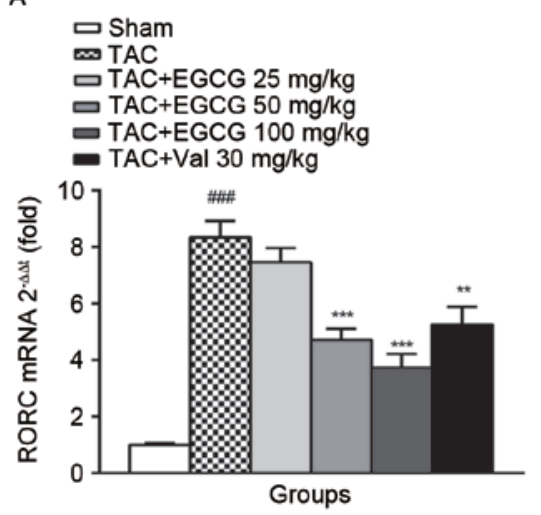

C

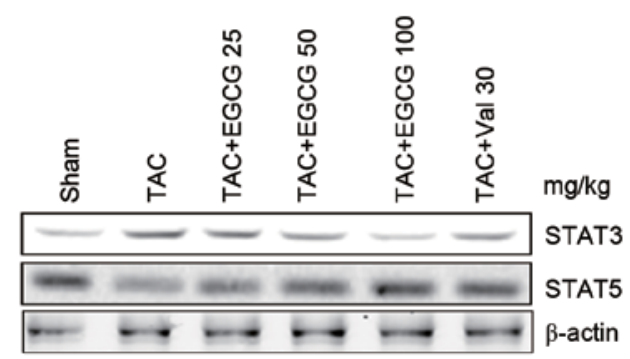

B

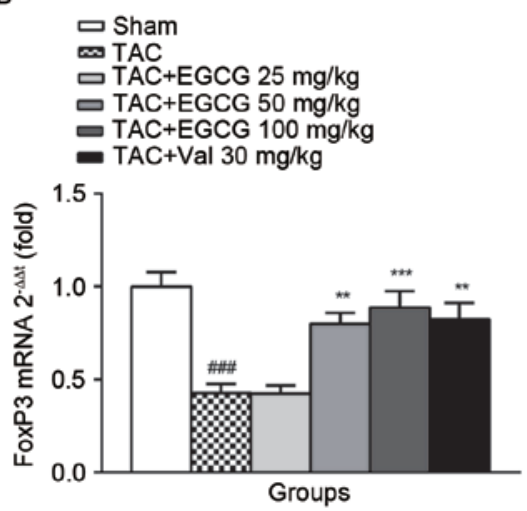

D

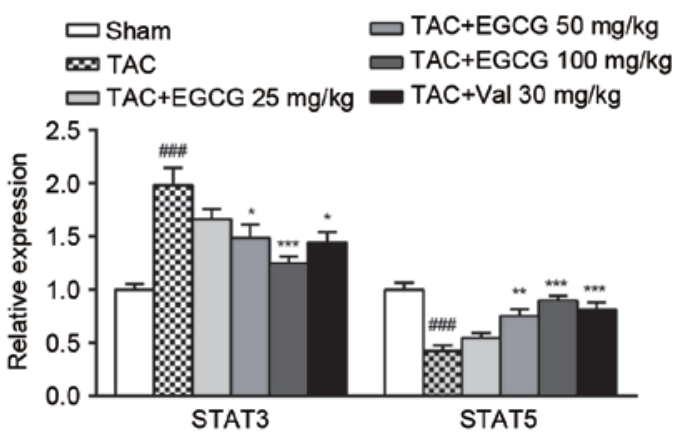

Figure 4. The effect of EGCG on the expressions of regulators for Th17 and Treg cells differentiation. (A) The level of RORC mRNA was found to be elevated in splenic lymphocytes of TAC rats using quantitative real-time RT-PCR (n=8), EGCG and Val treatment significantly decreased the overexpression of RORC. (B) FoxP3 expression was severely inhibited in splenic lymphocytes of TAC rats, and could be rescued by EGCG and Val administration (n=8). (C) Representative stripes for the expression of STAT3 and STAT5 in splenic lymphocytes. (D) The bands were quantified using Image J. EGCG and Val significantly restored the abnormal expressions of STAT3 and STAT5 in splenic lymphocytes of TAC rats $(\mathrm{n}=8)$. ${ }^{\# \# "} \mathrm{P}<0.001$ vs. sham-operated group; "P<0.05, ${ }^{* *} \mathrm{P}<0.01,{ }^{* * *} \mathrm{P}<0.001$ vs. TAC group. EGCG, Epigallocatechin gallate; TAC, transverse aortic constriction; Val, valsartan.

parameters ANP and BNP were elevated in TAC rats. In addition, Yu et al (27) reported that the ventricular stiffness is associated with $\mathrm{T}$ lymphocyte response in 2006 , indicating that cellular immunity may contribute to ECM production. Consistently, we found $\mathrm{T}$ cells were activated, and the ratio of Teff/Tnaive was up-regulated in TAC rats.

The Th1/Th2 populations which are characterized based on the unique patterns of surface markers, cytokine profiles, and immune functions are induced by specific ligands. In chronic alcohol-induced cardiac fibrosis, alcohol consumption contributes to the imbalance of Th1/Th2 subsets with an increased Th2 activity (4). Th1 and Th17 cytokine productions were increased, and Th2 cytokine was reduced in Chagas disease cardiomyopathy (28). Hypertensive rats induced by Ang II infusion were observed to have upregulation of IFN- $\gamma$ (Th1 cytokine) and downregulation of IL-4 (Th2 cytokine) (29). It was also reported that selectively induce Th1 leads to cardiac collagen deposit and collagen cross-linking, however, selectively induce $\mathrm{Th} 2$ subtype has the opposite effect in mice. As a result, the different Th cell phenotype distinctly influences the expression of pro-collagen and pro-MMP genes in cardiac fibroblasts (CFs), modulates the MMPs activity and collagen accumulation, contributes to the alteration of ECM composition, therefore, affects the diastolic function of the heart. Accordingly, regulation of the profile and function of Th lymphocyte could promote the adaptive ventricular remodeling in postmyocardial infarction and HF (6). In the present study, we found the mRNA level of T-bet in splenic lymphocytes of TAC rats was significantly increased, however, the level of GATA-3 mRNA was decreased, and the ratio of T-bet/GATA-3 was elevated. In parallel with the expression of the transcription factor, IFN- $\gamma$ (a Th1 cytokine) was elevated, while IL-10 (a Th2 cytokine) was diminished in the model rats. We illustrated that the imbalance of Th1/Th2 function exists in the cardiac remodeling process.

The homeostasis of $\mathrm{T}$ cells is regulated by two members of the common gamma chain family of cytokines, IL-7 and IL-15 (30). Interestingly, in the present study, we observed an upregulation of serum IL-15 and a downregulation of serum IL-7 in TAC rats. Literature also reported that plasma IL-7 level dropped in chronic heart failure patients (31). EGCG restored the homeostasis of $\mathrm{T}$ cells by rebalancing the expression of IL-7 and IL-15. IL-6 is considered an important cytokine in promoting the differentiation of Th17 cells from precursor Th cells. We revealed that in overload-induced cardiac remodeling rats, serum IL-6 production was increased significantly, resulting in the upregulation of both the percentage of Th17 and the level of IL-17. Previous studies have been conducted to exmine the role of IL-17 in inflammation-induced cardiac remodeling. It was reported that in IL-17-deficient mice, the interstitial myocardial fibrosis was attenuated, the expressions of MMP-2 and MMP-9 was reduced and the activity of gelatinase was inhibited. Anti-IL-17A monoclonal antibody could ameliorate both cardiac fibrosis and heart function when administered after the onset of myocarditis in BALB/c mice (32). Th17 cells and Treg cells develop reciprocally from 
A
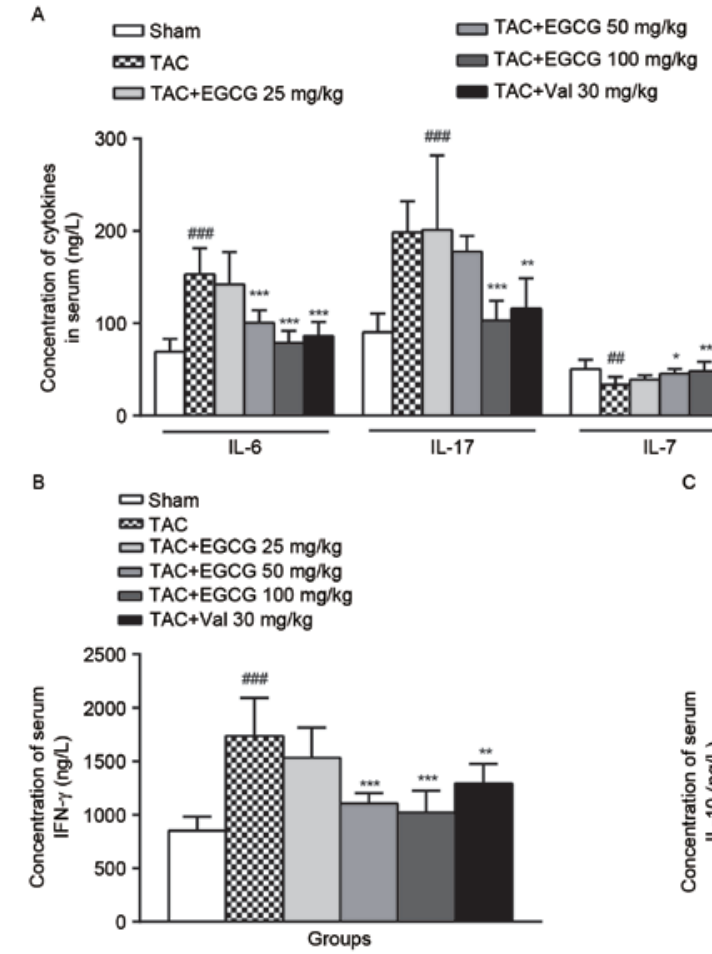

c

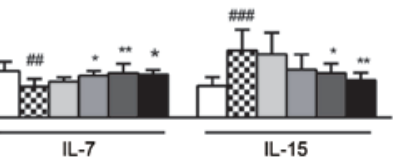

CSham
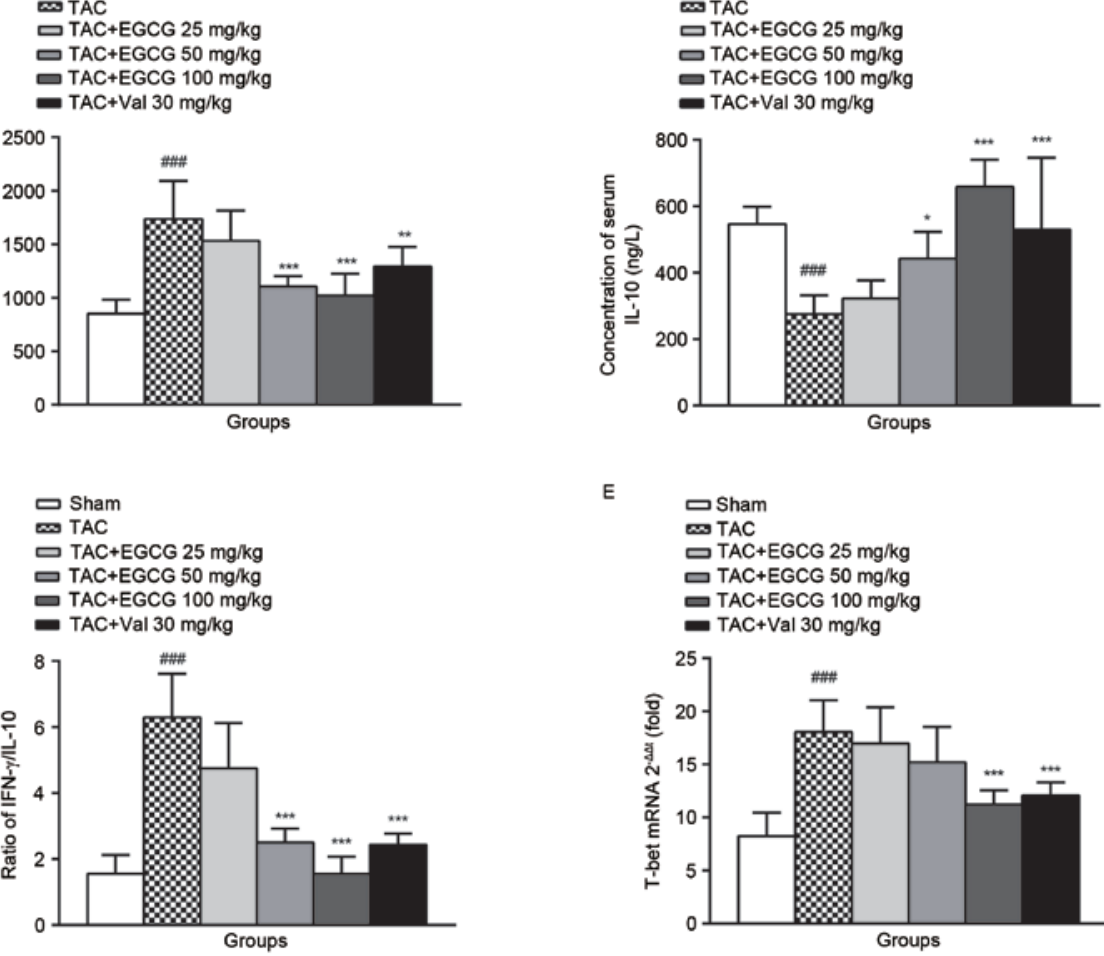

D Sham

TAC+EGCG $50 \mathrm{mg} / \mathrm{kg}$

- TAC+Val $30 \mathrm{mg} / \mathrm{k}$

E

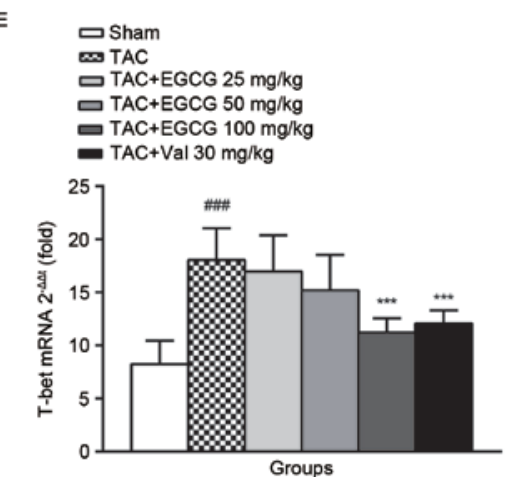

G
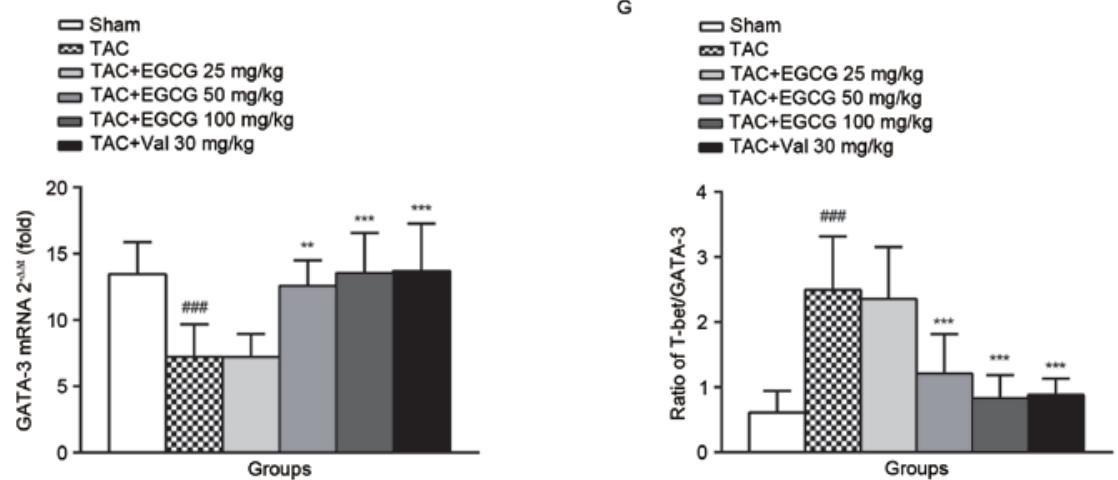

Figure 5. The effect of EGCG on the levels of serum Th1/Th2 cytokines. Serum levels of IL-6, IL-17, IFN- $\gamma$ and IL-10 were assessed by ELISA. (A) Both levels of IL-6 and IL-17 were increased in serum of cardiac remodeling rats, and were decreased by EGCG in a dose-dependent manner (n=8-10). (B) EGCG or Val inhibited the over expression of IFN- $\gamma(\mathrm{n}=8)$. (C) EGCG or Val slightly restored the level of serum IL-10. (D) EGCG or Val treatment significantly restored the unbalanced IFN- $\gamma /$ IL-10. (E) The levels of T-bet and GATA-3 mRNA were determined using quantitative real-time RT-PCR and the $2^{-\Delta \Delta C q}$ method was applied to assess the mRNA levels $(n=8)$. T-bet is a Th1-specific T box transcription factor. (F) GATA-3 is a critical regulator of Th2 polarization. (G) The ratio of T-bet/GATA-3. ${ }^{\# \#} \mathrm{P}<0.01,{ }^{\# \# \#} \mathrm{P}<0.001$ vs. sham-operated group; ${ }^{*} \mathrm{P}<0.05,{ }^{* *} \mathrm{P}<0.01,{ }^{* * *} \mathrm{P}<0.001$ vs. TAC group. EGCG, Epigallocatechin gallate; Val, valsartan; TAC, transverse aortic constriction.

naive Th cells, and exert opposite functions. The differentiation of Th17 depends on the activation of STAT3, while the development of Treg is mediated by the activation of STAT5 (23). The imbalance of Th17/Treg is an important feature of inflammation and autoimmune diseases (33). As expected, in TAC rats, we found a disequilibrated Th17/Treg balance with the expanded 
Th17 population and the shrinking Treg population compared with the sham-operated controls, indicating that the imbalance of Th17/Treg ratio potentially involved in the pathogenesis of cardiac ECM remodeling, and restoring this balance may be a promising therapeutic approach to attenuate cardiac remodeling.

Evidence shows that EGCG has multiple protective effects against many kinds of cardiovascular diseases, including cardiac fibrosis. However, how does ECCG prevent collagen deposit in the heart is still unknown. Cai and colleagues recently reported that reducing collagen production, fibronectin (FN) expression, and CFs proliferation by inhibiting $\mathrm{NF}-\kappa \mathrm{B}$ activation in rats challenged with AngII is the underlying molecular mechanism of EGCG (34). Besides cardiac fibrosis, EGCG is also a promising therapeutic drug for systemic sclerosis (SSc) induced oxidant stress and fibrosis (35).

In the present series of studies, we investigated whether the inhibitory effects of EGCG on collagen accumulation and heart remodeling were associated with the modulation of $\mathrm{T}$ lymphocytes subsets. As shown in the results, EGCG reduced total collagen content and MMPs activity in remodeled cardiac tissue indicating EGCG is a potential treatment for preventing cardiac remodeling in rats with overload induced cardiac ECM remodeling. We further showed that EGCG augmented Th2 cytokine production and diminished Th1 and Th17 cytokine production, that is in accordance with the modulation of the expression of T-bet and GATA-3, which are the master regulators for Th1 and Th2 differentiation respectively. It also decreased Th17 and increased Treg populations in the spleen through restoring the expression of Th17 and Treg downstream regulators STAT3 and STAT5. Moreover, EGCG inhibited T cell activation by rebalanced the population of Teff and Tnaive.

In conclusion, based on the current studies, EGCG is proved to be an effective therapeutic natural medicine in treating cardiac ECM remodeling and improving heart function, and restoring the balances of Th1/Th2, Th17/Treg, Teff/Tnaive and regaining the immune homeostasis are the presumed mechanisms of its action.

\section{Acknowledgements}

This study was supported by The National Natural Science Foundation of China (81202541), the Anhui Provincial Natural Science Foundation (1208085QH146 and 1408085MH173), the Grants for Scientific Research of BSKY (XJ201213), University Science Research Project of Anhui Province (KJ2017A176), Training Programme Foundation for the Young Talents of Higher Education (gxyqZD2017025), the Foundation for Young Academic Backbone of Anhui Medical University, the Grants for Young Talents of Anhui Medical University (2013), Young Outstanding Doctor Research Program, Anhui Provincial Hospital (2015).

\section{References}

1. Morita $\mathrm{H}$ and Komuro I: Periostin isoforms and cardiac remodeling after myocardial infarction: Is the dispute settled? Hypertension 67: 504-505, 2016.

2. Xu X, Pang J, Chen Y, Bucala R, Zhang Y and Ren J: Macrophage migration inhibitory factor (MIF) deficiency exacerbates aging-induced cardiac remodeling and dysfunction despite improved inflammation: Role of autophagy regulation. Sci Rep 6: 22488, 2016.
3. Melendez GC, McLarty JL, Levick SP, Du Y, Janicki JS and Brower GL: Interleukin 6 mediates myocardial fibrosis, concentric hypertrophy, and diastolic dysfunction in rats. Hypertension 56: 225-231, 2010.

4. Liu W, Li J, Tian W, Xu T and Zhang Z: Chronic alcohol consumption induces cardiac remodeling in mice from Th1 or Th2 background. Exp Mol Pathol 91: 761-767, 2011.

5. Kwon WY, Cha HN, Heo JY, Choi JH, Jang BI, Lee IK and Park SY: Interleukin-10 deficiency aggravates angiotensin II-induced cardiac remodeling in mice. Life Sci 146: 214-221, 2016.

6. Yu Q, Vazquez R, Zabadi S, Watson RR and Larson DF: T-lymphocytes mediate left ventricular fibrillar collagen cross-linking and diastolic dysfunction in mice. Matrix Biol 29: 511-518, 2010.

7. Yang CS, Chen G and Wu Q: Recent scientific studies of a traditional chinese medicine, tea, on prevention of chronic diseases. J Tradit Complement Med 4: 17-23, 2014.

8. Liu S, Xu ZL, Sun L, Liu Y, Li CC, Li HM, Zhang W, Li CJ and Qin W: (-)-Epigallocatechin-3-gallate induces apoptosis in human pancreatic cancer cells via PTEN. Mol Med Rep 14: 599-605, 2016.

9. Zeng X and Tan X: Epigallocatechin-3-gallate and zinc provide anti-apoptotic protection against hypoxia/reoxygenation injury in H9c2 rat cardiac myoblast cells. Mol Med Rep 12: 1850-1856, 2015.

10. Tang J, Zheng JS, Fang L, Jin Y, Cai W and Li D: Tea consumption and mortality of all cancers, CVD and all causes: A meta-analysis of eighteen prospective cohort studies. Br J Nutr 114: 673-683, 2015 .

11. Xuan F and Jian J: Epigallocatechin gallate exerts protective effects against myocardial ischemia/reperfusion injury through the PI3K/Akt pathway-mediated inhibition of apoptosis and the restoration of the autophagic flux. Int J Mol Med 38: 328-336, 2016.

12. Tian C, Huang Q, Yang L, Légaré S, Angileri F, Yang H, Li X, Min $\mathrm{X}$, Zhang $\mathrm{C}$, $\mathrm{Xu} \mathrm{C}$, et al: Green tea consumption is associated with reduced incident CHD and improved CHD-related biomarkers in the Dongfeng-Tongji cohort. Sci Rep 6: 24353, 2016.

13. Cai Y, He SQ, Hong HQ, Cai YP, Zhao L and Zhang M: High doses of (-)-epigallocatechin-3-gallate from green tea induces cardiac fibrosis in mice. Biotechnol Lett 37: 2371-2377, 2015.

14. Han YS, Lan L, Chu J, Kang WQ and Ge ZM: Epigallocatechin gallate attenuated the activation of rat cardiac fibroblasts induced by angiotensin II via regulating $\beta$-arrestin1. Cell Physiol Biochem 31: 338-346, 2013.

15. Kim YH, Won YS, Yang X, Kumazoe M, Yamashita S, Hara A, Takagaki A, Goto K, Nanjo F and Tachibana H: Green tea catechin metabolites exert immunoregulatory effects on CD4 (+) $\mathrm{T}$ cell and natural killer cell activities. J Agric Food Chem 64: 3591-3597, 2016.

16. Li X, Zhang L and Liang J: Unraveling the expression profiles of long noncoding RNAs in rat cardiac hypertrophy and functions of 1ncRNA BC088254 in cardiac hypertrophy induced by transverse aortic constriction. Cardiology 134: 84-98, 2016.

17. Guo K, Lan CZ, Yu TT, Huang LL, Wang XH, Pan C and Gao S: Effects of Xin-Ji-Er-Kang formula on 2K1C-induced hypertension and cardiovascular remodeling in rats. J Ethnopharmacol 155: 1227-1235, 2014

18. Wen Y, Zeng Z, Gui C, Li L and Li W: Changes in the expression of Th17 cell-associated cytokines in the development of rheumatic heart disease. Cardiovasc Pathol 24: 382-387, 2015.

19. Huang B, Wang QT, Song SS, Wu YJ, Ma YK, Zhang LL, Chen JY, Wu HX, Jiang L and Wei W: Combined use of etanercept and MTX restores CD4*/CD8* ratio and Tregs in spleen and thymus in collagen-induced arthritis. Inflamm Res 61: 1229-1239, 2012

20. Chen J, Feng X and Huang Q: Modulation of T-Bet and GATA-3 expression in experimental autoimmune thyroiditis rats through ginsenoside treatment. Endocr Res 41: 28-33, 2016.

21. Wang QT, Zhang LL, Wu HX and Wei W: The expression change of $\beta$-arrestins in fibroblast-like synoviocytes from rats with collagen-induced arthritis and the effect of total glucosides of paeony. J Ethnopharmacol 133: 511-516, 2011.

22. Terrazas C, Varikuti S, Kimble J, Moretti E, Boyaka PN and Satoskar AR: IL-17A promotes susceptibility during experimental visceral leishmaniasis caused by Leishmania donovani. FASEB J 30: 1135-1143, 2016. 
23. Lee YJ, Hyung KE, Yoo JS, Jang YW, Kim SJ, Lee DI, Lee SJ, Park SY, Jeong JH and Hwang KW: Effects of exposure to extremely low-frequency electromagnetic fields on the differentiation of Th17 T cells and regulatory T cells. Gen Physiol Biophys 35: 487-495, 2016.

24. Stellato C, Gubin MM, Magee JD, Fang X, Fan J, Tartar DM, Chen J, Dahm GM, Calaluce R, Mori F, et al: Coordinate regulation of GATA-3 and Th2 cytokine gene expression by the RNA-binding protein HuR. J Immunol 187: 441-449, 2011.

25. Moore-Morris T, Guimarães-Camboa N, Yutzey KE, Pucéat M and Evans SM: Cardiac fibroblasts: From development to heart failure. J Mol Med (Berl) 93: 823-830, 2015.

26. Badenhorst D, Maseko M, Tsotetsi OJ, Naidoo A, Brooksbank R, Norton GR and Woodiwiss AJ: Cross-linking influences the impact of quantitative changes in myocardial collagen on cardiac stiffness and remodelling in hypertension in rats. Cardiovase Res 57: 632-641, 2003.

27. Yu Q, Horak K and Larson DF: Role of T lymphocytes in hypertension-induced cardiac extracellular matrix remodeling. Hypertension 48: 98-104, 2006.

28. Nogueira LG, Santos RH, Fiorelli AI, Mairena EC, Benvenuti LA, Bocchi EA, Stolf NA, Kalil J and Cunha-Neto E: Myocardial gene expression of T-bet, GATA-3, Ror- $\gamma$ t, FoxP3, and hallmark cytokines in chronic Chagas disease cardiomyopathy: An essentially unopposed TH1-type response. Mediators Inflamm 2014: 914326, 2014.
29. Shao J, Nangaku M, Miyata T, Inagi R, Yamada K, Kurokawa K and Fujita T: Imbalance of T-cell subsets in angiotensin II-infused hypertensive rats with kidney injury. Hypertension 42: 31-38, 2003.

30. Jung YW, Kim HG, Perry CJ and Kaech SM: CCR7 expression alters memory CD8 T-cell homeostasis by regulating occupancy in IL-7- and IL-15-dependent niches. Proc Natl Acad Sci USA 113: 8278-8283, 2016

31. Cappuzzello C, Di Vito L, Melchionna R, Melillo G, Silvestri L, Cesareo E, Crea F, Liuzzo G, Facchiano A, Capogrossi MC and Napolitano M: Increase of plasma IL-9 and decrease of plasma IL-5, IL-7 and IFN- $\gamma$ in patients with chronic heart failure. J Transl Med 9: 28, 2011.

32. Machino-Ohtsuka T, Tajiri K, Kimura T, Sakai S, Sato A, Yoshida T, Hiroe M, Yasutomi Y, Aonuma K and Imanaka-Yoshida $\mathrm{K}$ : Tenascin- $\mathrm{C}$ aggravates autoimmune myocarditis via dendritic cell activation and Th17 cell differentiation. J Am Heart Assoc 3: e001052, 2014.

33. Lim SM, Kang GD, Jeong JJ, Choi HS and Kim DH Neomangiferin modulates the Th17/Treg balance and ameliorates colitis in mice. Phytomedicine 23: 131-140, 2016.

34. Cai Y, Yu SS, Chen TT, Gao S, Geng B, Yu Y, Ye JT and Liu PQ: EGCG inhibits CTGF expression via blocking NF- $\kappa$ B activation in cardiac fibroblast. Phytomedicine 20: 106-113, 2013.

35. Dooley A, Bruckdorfer KR and Abraham DJ: Modulation of fibrosis in systemic sclerosis by nitric oxide and antioxidants. Cardiol Res Pract 2012: 521958, 2012. 